\title{
Novel Spin Dynamics in a Josephson Junction
}

\author{
Jian-Xin Zhu, ${ }^{1}$ Zohar Nussinov, ${ }^{1}$ Alexander Shnirman, ${ }^{1,2}$ Alexander V. Balatsky ${ }^{1}$ \\ 1 Theoretical Division, Los Alamos National Laboratory, Los Alamos, New Mexico 87545 and \\ 2 Institut für Theoretische Festkörperphysik, Universität Karlsruhe, D-76128 Karlsruhe, Germany
}

\begin{abstract}
We address the dynamics of a single spin embedded in the tunneling barrier between two superconductors. As a consequence of pair correlations in the superconducting state, the spin displays a rich and unusual dynamics. To properly describe the time evolution of the spin we find the generalized Wess-Zumino-Witten-Novikov term in the effective action for the spin on the Keldysh contour. The superconducting correlations lead to an effective spin action which is non-local in time leading to unconventional precessions. Our predictions might be directly tested for macroscopic spin clusters.
\end{abstract}

PACS numbers: 74.50.+r, 75.20.Hr, 73.40.Gk

There is a growing interest in a number of techniques that allow one to detect and manipulate a single spin in the solid state. A partial list includes optical detection of electron spin resonance (ESR) in a single molecule [1], tunneling through a quantum dot 2], and, more recently, ESR-scanning tunneling microscopy (ESR-STM) technique 3, 4]. Interest in ESR-STM lies in the possibility to detect and manipulate a single spin [5, 6], which is crucial in spintronics and quantum information processing. In a similar investigation much work has been done to address the coupling, feedback effects, and decoherence in a coupled electronic-vibrational systems, such as nanomechanical oscillators and local vibrational modes 7]. In a previous publication, two of us studied the effect of a precessing spin on the supercurrent [8]. In the present Letter, we complement this earlier study and focus on how a spin is affected by a supercurrent. Our major finding is that the spin dynamics is no longer that of simple precession. We provide a direct prediction of the expected spin dynamics. This unusual spin dynamics characterized by longitudinal oscillations is caused by coupling to a Josephson current. Due to spin normalization, these novel spin precessions do not lead to any changes in the Josephson current. Keldysh contour calculations illustrate that a non-local in time single fermion action is also found in situations wherein the single spin cluster is replaced by an Anderson impurity [9]. As well known, in the limit of small hopping amplitudes to and from an Anderson impurity, the impurity attains a Kondo like character much like that of the single spin which is the focus of our attention. Here we consider the origin of this rather generic non-locality in time present in the dynamics of a Josephson junction. Our primary focus will be on larger spin clusters for which direct measurements can be made to probe this novel spin dynamics.

The model system under consideration is illustrated in Fig. [1 It consists of two ideal superconducting leads coupled to each other by a single magnetic spin. In the presence of a magnetic field, the spin precesses around the field direction. We neglect the interaction of the spin with two superconducting leads. The Hamiltonian for the Josephson junction can be written as [10, 11]:

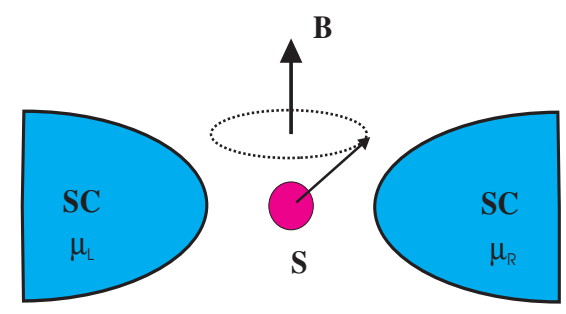

FIG. 1: Magnetic spin coupled to two superconducting leads. In the presence of a magnetic field $\mathbf{B}$, the spin precesses around the field direction. As we illustrate here, in a Josephson junction the standard precession is accompanied by a polar motion leading to a full blown rigid body like dynamics.

$H=H_{L}+H_{R}+H_{T}$. The first two terms are the Hamiltonians for electrons in the left and right superconducting leads $H_{L(R)}=\sum_{k(p) ; \sigma} \epsilon_{k(p)} c_{k(p), \sigma}^{\dagger} c_{k(p), \sigma}+$ $\frac{1}{2} \sum_{k(p) ; \sigma, \sigma^{\prime}}\left[\Delta_{\sigma \sigma^{\prime}}(k(p)) c_{k(p), \sigma}^{\dagger} c_{-k(-p), \sigma^{\prime}}^{\dagger}+\right.$ H.c. $]$, where we have denoted the electron creation (annihilation) operators in the left $(\mathrm{L})$ lead by $c_{k \sigma}^{\dagger}\left(c_{k \sigma}\right)$ while those in the right $(\mathrm{R})$ lead by $c_{p \sigma}^{\dagger}\left(c_{p \sigma}\right)$. The quantities $k(p)$ are momenta, $\sigma$ the spin index, and $\epsilon_{k(p), \sigma}, \Delta_{\sigma \sigma^{\prime}}(k(p))$ are, respectively, the single particle energies of conduction electrons, and the pair potential in the leads. In this work, we consider $s$-wave pairing symmetry in the superconducting leads. The two leads are weakly coupled via the tunneling Hamiltonian: $H_{T}=\sum_{k, p ; \sigma, \sigma^{\prime}}\left[T_{\sigma \sigma^{\prime}}(k, p) c_{k \sigma}^{\dagger} c_{p \sigma^{\prime}}+\right.$ H.c. $]$, where the matrix element $T_{\sigma \sigma^{\prime}}(k, p)$ transfer electrons through an insulating barrier. When a spin is embedded in the tunneling barrier, the tunneling matrix becomes a spin operator $[\underline{6}]: \hat{T}=T_{0} \delta_{\sigma \sigma^{\prime}}+T_{1} \mathbf{S} \cdot \boldsymbol{\sigma}_{\sigma \sigma^{\prime}}$, where $T_{0}$ is a spin-independent tunneling matrix element and $T_{1}$ is a spin-dependent matrix element originating from the direct exchange coupling $J$ of the conduction electron to the localized spin $\mathbf{S}$. We take both to be momentum independent. This is not a crucial assumption and is merely introduced to simplify notations. Typically, from the expansion of the work function for tunneling, $\frac{T_{1}}{T_{0}} \sim J / U$, where $U$ is a spin-independent tunneling barrier [8]. We further allow a weak external magnetic field $B \sim 10^{2}$ Gauss. Such a small field will not influence the 
superconducting state and we may ignore its effect on the leads. The Josephson junction with the spin has two time scales: (i) The Larmor precession frequency of the spin $\omega_{L}=g \mu_{B} B$, where $g, \mu_{B}$ are the gyromagnetic ratio and Bohr magneton of the conduction electron, respectively. (ii) The frequency $\omega_{J}=2 e V$ characterizing the Josephson effect when an external voltage $V$ is applied.

We now derive the effective action via the Keldysh technique. If all external fields are the same on both forward and backward branches of the Keldysh contour $(C)$ then $\mathcal{Z}=\operatorname{Tr} T_{C} \exp \left[-i \oint_{C} d t H_{T}(t)\right]=1$, where the trace is over both the electron and the spin degrees of freedom. We first take a partial trace in $\mathcal{Z}$ over the lead fermions (the bath) to obtain an effective spin action. The Josephson contribution to the resulting spin action reads $i \delta \mathcal{S}=-\frac{1}{2} \oint_{C} d t \oint_{C} d t^{\prime}\left\langle T_{C} H_{T}(\mathbf{S}(t), t) H_{T}\left(\mathbf{S}\left(t^{\prime}\right), t^{\prime}\right)\right\rangle$, much in the spirit of Refs. [12].

For brevity, we set $A_{\sigma, \sigma^{\prime}} \equiv \sum_{k, p} c_{k \sigma}^{\dagger} c_{p \sigma^{\prime}}$. The tunneling Hamiltonian of a phase (voltage) biased junction

$$
\begin{aligned}
H_{T}= & {\left[T_{0} \delta_{\sigma \sigma^{\prime}}+T_{1} \mathbf{S} \cdot \boldsymbol{\sigma}_{\sigma \sigma^{\prime}}\right] } \\
& \times\left(A_{\sigma \sigma^{\prime}} \exp (i \phi / 2)+A_{\sigma \sigma^{\prime}}^{\dagger} \exp (-i \phi / 2)\right) .
\end{aligned}
$$

In the presence of a dc voltage bias, $\phi=2 \mathrm{eVt}$. As $\phi$ is treated classically (i.e. $\phi$ is the same on the upper and the lower branches of the Keldysh contour), the contribution $\propto T_{0}^{2}$ to $\delta \mathcal{S}$ vanishes. The mixed contribution $\propto T_{0} T_{1}$ vanishes due to the singlet spin structure of the s-wave superconductor. The only surviving contribution reads

$$
\begin{gathered}
i \delta \mathcal{S}=-\frac{T_{1}^{2}}{2} \oint_{C} d t \oint_{C} d t^{\prime}\left[\mathbf{S}(t) \cdot \boldsymbol{\sigma}_{\alpha \beta}\right]\left[\mathbf{S}\left(t^{\prime}\right) \cdot \boldsymbol{\sigma}_{\delta \gamma}\right] \times \\
\left(\left\langle T_{C} A_{\alpha \beta}(t) A_{\delta \gamma}\left(t^{\prime}\right)\right\rangle e^{i \frac{\phi(t)+\phi\left(t^{\prime}\right)}{2}}+\left(A, \phi \rightarrow A^{\dagger},-\phi\right)\right)(2)
\end{gathered}
$$

where we keep only the Josephson (off-diagonal) terms. The spin structure simplifies for the s-wave case:

$$
i \delta \mathcal{S}=T_{1}^{2} \oint_{C} d t \oint_{C} d t^{\prime}\left[\mathbf{S}(t) \cdot \mathbf{S}\left(t^{\prime}\right)\right]\left[i D\left(t, t^{\prime}\right)\right]
$$

where $i D\left(t, t^{\prime}\right) \equiv\left\langle T_{C} A_{\uparrow \uparrow}(t) A_{\downarrow \downarrow}\left(t^{\prime}\right)\right\rangle e^{i \frac{\phi(t)+\phi\left(t^{\prime}\right)}{2}}+(A, \phi \rightarrow$ $\left.A^{\dagger},-\phi\right)$. Next, we perform the standard Keldysh manipulations, defining upper and lower spin fields $\mathbf{S}^{u, l}$ residing on the forward/backward contours and reducing the time ordered integral over Keldysh contour to the integral over forward running time at the cost of making the Green's function $G$ a $2 \times 2$ matrix. Finally, after a rotation to the classical and quantum components

$$
\mathbf{S}_{1} \equiv\left(\mathbf{S}^{u}+\mathbf{S}^{l}\right) / 2, \quad \mathbf{S}_{2} \equiv \mathbf{S}^{u}-\mathbf{S}^{l}, \quad \mathbf{S}_{1} \cdot \mathbf{S}_{2}=0,
$$

we obtain $i \delta \mathcal{S}=i \mathcal{S}_{R}+\mathcal{S}_{I}$, where

$$
i \mathcal{S}_{R}=\iint d t d t^{\prime} \mathbf{S}_{2}(t) \cdot \mathbf{S}_{1}\left(t^{\prime}\right)\left[i K_{12}\left(t, t^{\prime}\right)\right] .
$$

with $K_{12}\left(t, t^{\prime}\right)=T_{1}^{2}\left[D^{R}\left(t, t^{\prime}\right)+D^{A}\left(t^{\prime}, t\right)\right]$, and

$$
\mathcal{S}_{I}=\frac{1}{2} \iint d t d t^{\prime} \mathbf{S}_{2}(t) \cdot \mathbf{S}_{2}\left(t^{\prime}\right)\left[i K_{22}\left(t, t^{\prime}\right)\right],
$$

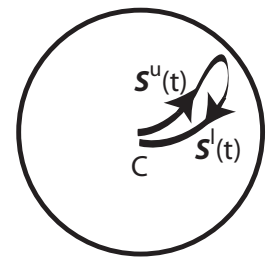

FIG. 2: The unit sphere for the vectors $\mathbf{n}^{u, l}(t)$ is shown. The contour $C$ is the Keldysh contour for the forward $(u)$ and backward $(l)$ evolution. To properly describe the spin on this closed contour we analyze the WZWN term, see Eq. (13). For clarity, we draw a small piece of the closed trajectories.

with $K_{22}\left(t, t^{\prime}\right)=T_{1}^{2} D^{K}\left(t, t^{\prime}\right)$. The retarded $(\mathrm{R})$ and Keldysh $(\mathrm{K})$ components are defined via

$$
\begin{aligned}
i D^{R}\left(t, t^{\prime}\right)= & \Theta\left(t-t^{\prime}\right) \\
& \times\left\langle\left[A_{\uparrow \uparrow}(t), A_{\downarrow \downarrow}\left(t^{\prime}\right)\right]_{-}\right\rangle e^{i \frac{\phi(t)+\phi\left(t^{\prime}\right)}{2}}-\text { c.c. } \\
i D^{K}\left(t, t^{\prime}\right)= & \left\langle\left\{A(t)_{\uparrow \uparrow}, A_{\downarrow \downarrow}\left(t^{\prime}\right)\right\}_{+}\right\rangle e^{i \frac{\phi(t)+\phi\left(t^{\prime}\right)}{2}}+\text { c.c. }
\end{aligned}
$$

The advanced component in our case is simply $i D^{A}\left(t, t^{\prime}\right)=i D^{R}\left(t^{\prime}, t\right)$. The kernels $K_{12}$ and $K_{22}$ are readily calculated at $T=0$ from

$$
\left\langle A(t)_{\uparrow \uparrow} A_{\downarrow \downarrow}\left(t^{\prime}\right)\right\rangle=\sum_{k, p} \frac{|\Delta|^{2}}{4 E_{k} E_{p}} e^{-i\left(E_{k}+E_{p}\right)\left(t-t^{\prime}\right)},
$$

where $E_{k(p)}=\sqrt{\left(\epsilon_{k(p)}-E_{F}\right)^{2}+|\Delta|^{2}}$. As only frequencies higher than $2 \Delta$ are present in (9), $S_{I}$ (Eq. (6) ) vanishes for slow fluctuations of $\mathbf{S}_{2}$ unlike the real part of the action $S_{R}$ (due to the presence of $\Theta$ function in Eq. (7)).

To properly describe the dynamics of the spin fields $\mathbf{S}_{1}$ and $\mathbf{S}_{2}$, we employ the path integral representation for the spin fields [13]. The action for a free spin consists of two terms $\mathcal{S}_{0}=g \mu_{B} \oint_{C} d t \mathbf{B} \cdot \mathbf{S}+\mathcal{S}_{W Z W N}$. The second, Wess-Zumino-Witten-Novikov (WZWN), term describes the Berry phase accumulated by the spin as a result of motion of the spin on the sphere [13]. We generalize this action for nonequlibrium dynamics within the Keldysh contour formalism (Fig. 2). We write the WZWN term on both forward and backward contours, and consequently analyze matters in terms of $\mathbf{S}_{1}$ and $\mathbf{S}_{2}$,

$$
\begin{array}{r}
i \mathcal{S}_{W Z W N}=\frac{i}{S^{2}} \int_{0}^{1} d \tau \int d t\left[\mathbf { S } ^ { u } ( t , \tau ) \cdot \left(\partial_{\tau} \mathbf{S}^{u}(t, \tau)\right.\right. \\
\left.\left.\times \partial_{t} \mathbf{S}^{u}(t, \tau)\right)-(u \rightarrow l)\right]
\end{array}
$$

The relative minus sign stems from the backward time ordering on the return part of $C$. The spins $\mathbf{S}^{u, l}=S \mathbf{n}^{u, l}$ with $S$ the magnitude of the spin and $\mathbf{n}^{u, l}$ a unit vector field. The additional integral over $\tau$ permits us to express the action in a local form. At $\tau=0$ we set the spin to point along the $z$ direction at all times; at $\tau=1$ the spin field corresponds to the physical configurations. Each of the individual WZWN phases (for both 
the forward $(u)$ and backward $(l)$ branches) is the spin magnitude $(S)$ multiplied by the areas spanned by the trajectories $\mathbf{n}^{u, l}(t)$ on the unit sphere. The WZWN term contains odd powers of $\mathbf{S}_{2}$. Insofar as the WZWN term of Eq. (10) is concerned, the standard Keldysh transformation to the two classical and quantum fields, $\mathbf{S}_{1}$ and $\mathbf{S}_{2}$, mirrors the decomposition of the spin in an antiferromagnet (AF) to the two orthogonal slow and fast fields 14]. The difference between the two individual WZWN terms in Eq.110) is the area spanned between the forward and backward time trajectories. The magnitude of this area traced between times $t$ and $t+\delta t$ is

$$
\delta \mathcal{S}_{W Z W N}=S(\delta t) \delta \mathbf{n} \cdot\left(\mathbf{n} \times \partial_{t} \mathbf{n}\right) .
$$

Here, the variation between the forward and backward trajectory at a given instant of time is $\delta \mathbf{n}=\mathbf{S}_{2} / S$. In Eq. (11), we note that for small variations between the forward and backward trajectories, $\mathbf{n}=\mathbf{S}_{1} / S$. The WZWN action on the Keldysh loop may be expressed as

$$
\mathcal{S}_{W Z W N}=\frac{1}{S^{2}} \int d t \mathbf{S}_{2} \cdot\left(\mathbf{S}_{1} \times \partial_{t} \mathbf{S}_{1}\right) .
$$

The real part of the total action, $\mathcal{S}_{c l} \equiv \mathcal{S}_{0}+\mathcal{S}_{R}$, determines the quasi-classical equation of motion. The imaginary part of the action $\mathcal{S}_{I}$ is usually responsible for the Langevin stochastic term. In our case, however, the Langevin term is suppressed at frequencies much lower than $\Delta$. The fluctuations of $\mathbf{S}_{2}$ are not suppressed by the bath and no dissipation appears. The only reason to consider small fluctuations of $\mathbf{S}_{2}$ is the dominance of the quasi-classical trajectories determined by $\mathcal{S}_{c l}$. Thus our analysis applies for large spins. The classical action

$$
\begin{gathered}
\mathcal{S}_{c l}=\mathcal{S}_{W Z W N}+g \mu_{B} \int d t \mathbf{B} \cdot \mathbf{S}_{2} \\
+\int d t \int d t^{\prime} K_{12}\left(t, t^{\prime}\right) \mathbf{S}_{2}(t) \cdot \mathbf{S}_{1}\left(t^{\prime}\right) .
\end{gathered}
$$

As the spin dynamics is much slower as compared to electronic processes, we set $\mathbf{S}_{1}\left(t^{\prime}\right) \simeq \mathbf{S}_{1}(t)+\left(t^{\prime}-t\right) d \mathbf{S}_{1} / d t$. The variational equations $\delta S_{c l} / \delta \mathbf{S}_{\mathbf{2}}(t)=0$ imply

$$
\frac{d \mathbf{n}}{d t}=\alpha \mathbf{n} \times \frac{d \mathbf{n}}{d t} \sin \omega_{J} t+g \mu_{B} \mathbf{n} \times \mathbf{B},
$$

where henceforth we denote $\mathbf{S}_{1}$ by $\mathbf{S}=S \mathbf{n}$, and $\alpha=$ $S \sum_{k, p} \frac{|\Delta|^{2}\left|T_{1}\right|^{2}}{E_{k} E_{p}}\left[\left(E_{k}+E_{p}-e V\right)^{-2}-\left(E_{k}+E_{p}+e V\right)^{-2}\right]$.

The non-dissipative term proportional to $\alpha$ in Eq. (14) arises from superconducting retardations. Both $\mathbf{S} \times \frac{d \mathbf{S}}{d t}$ and $I \sim \sin \omega_{J} t$ are odd in time and their product is allowed in the equation of motion. Due to the spectral gap, dissipation is faint at low temperatures and frequencies.

The classical equation of motion, Eq. (14), implies that $\mathbf{n} \cdot d \mathbf{n} / d t=0$, as it must to be consistent with the parameterization of the spin on the sphere, $S \mathbf{n}=$
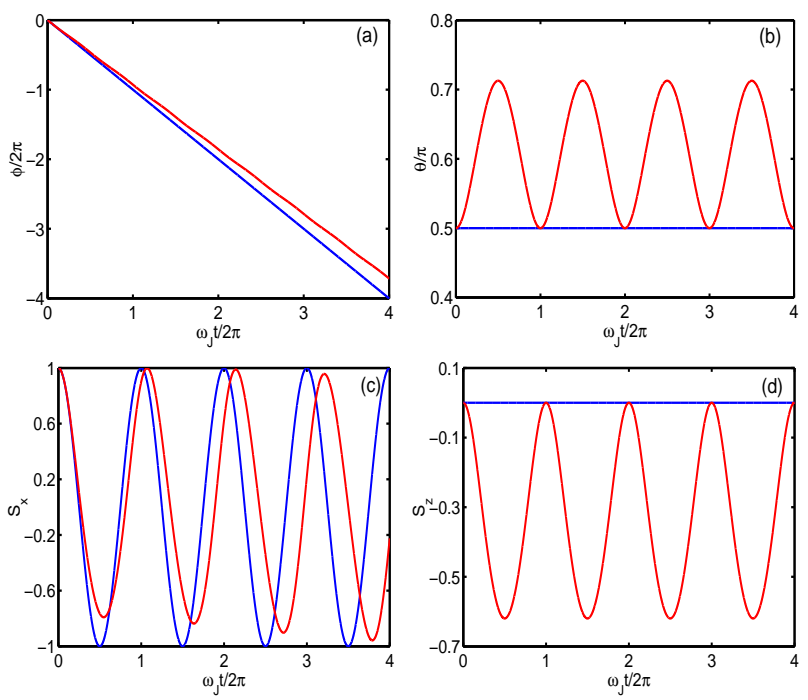

FIG. 3: The spin dynamics. Top panels: the azimuthal $(\phi)$ and polar $(\theta)$ angles as a function of time for an exaggerated $\alpha=0.4$ (red) versus the standard precessions in the absence of the coupling ( $\alpha=0$ in blue). Note that unlike the standard case, the semi-classical spin trajectory is not confined to planar motions. The polar angle oscillates between its two extreme values $\theta_{1}$ and $\theta_{2}$. In the bottom panels we display $\left\langle S_{x}(t)\right\rangle$ and $\left\langle S_{z}(t)\right\rangle$. Here we take $\omega_{L}=\omega_{J}$.

$S(\sin \theta \cos \phi, \sin \theta \sin \phi, \cos \theta)$. Orienting the $z$-axis along the external magnetic field $\mathbf{B}$, Eq. (14) reads

$$
\begin{aligned}
& \frac{d \phi}{d t}=-\frac{\omega_{L}}{1+\alpha^{2} \sin ^{2}\left(\omega_{J} t\right)}, \\
& \frac{d \theta}{d t}=-\alpha \frac{d \phi}{d t} \sin \theta \sin \omega_{J} t .
\end{aligned}
$$

For a spin initially oriented at an angle $\theta_{0}$ relative to $\mathbf{B}$,

$$
\begin{aligned}
& \phi(t)=-\frac{\omega_{L}}{\omega_{J} \sqrt{1+\alpha^{2}}} \tan ^{-1}\left[\sqrt{1+\alpha^{2}} \tan \left(\omega_{J} t\right)\right], \\
& \theta(t)=2 \tan ^{-1}\left(\left[\frac{\left(1-c \cos \left(\omega_{J} t\right)\right)(1+c)}{\left(1+c \cos \left(\omega_{J} t\right)\right)(1-c)}\right]^{\gamma} \tan \frac{\theta_{0}}{2}\right),
\end{aligned}
$$

with $c=|\alpha| / \sqrt{1+\alpha^{2}}$ and $\gamma=-\alpha \omega_{L} / 2 \omega_{J} c$. For $\alpha \ll 1$, $\phi \simeq-\omega_{L} t$ and $\theta \simeq \theta_{0}-\alpha\left(\omega_{L} / \omega_{J}\right) \sin \theta_{0} \cos \omega_{J} t$. Canonically, whenever a single spin is subjected to a uniform magnetic field, the spin precesses azimuthally with a frequency $\omega_{L}$. In a Josephson junction, however, the spin exhibits additional polar $(\theta)$ displacements. The resulting dynamics may be likened to that of a rotating rigid top. The Josephson current leads to a full non-planar gyroscopic motion (nutation) of the spin much like that generated by applied "torques" on a mechanical top. When $\mathbf{B}=0$, the solutions do not allow for any spin dynamics. The possibility remains, nonetheless, that coupling to random magnetic field (bath) will have a nontrivial effect on the spin in presence of a Josephson current. In Fig. 3. we display the resulting dynamics for the spin. 


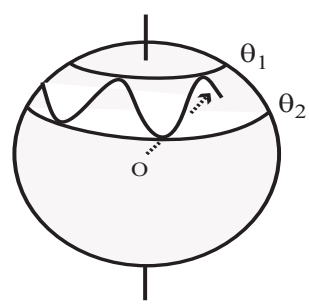

FIG. 4: The resulting spin motion on the unit sphere in the general case. As in the motion of classical spinning top, the spin exhibits undulations along the polar direction.

A schematic of the generic spin motion is displayed in Fig. 4 Similar to a classical spinning top, the spin wobbles along the polar direction in addition to azimuthal rotations. Similar dynamics is even expected for a quantum $S=1 / 2$. In a spin coherent state path integral, the Schrodinger equation is essentially classical.

The Josephson current in the presence of a precessing spin has been discussed in [8]. Within our approximation, the terms in the action that may affect the current are proportional to $\mathbf{S}^{2}=1$ and $\mathbf{S} \cdot \frac{\partial \mathbf{S}}{\partial t}=0$. Consequently, the Josephson current remains unmodulated. Nevertheless, we may directly monitor the spin dynamics by SQUID measurements. The spin motion generates a time dependent magnetic field, $\delta \mathbf{B}(\mathbf{r}, t)=$ $\frac{\mu_{0}}{4 \pi}\left[3 \mathbf{r}(\mathbf{r} \cdot \mathbf{m}(t))-r^{2} \mathbf{m}(t)\right] / r^{5}$, superimposed against the constant external field $\mathbf{B}$. Here, $\mathbf{r}$ is the position from the spin and the magnetic moment $\mathbf{m}(t)=g \mu_{B} S \mathbf{n}(t)$. For a ferromagnetic cluster of spin $S=100$, a detectable field $\delta B \sim 10^{-10}$ Tesla appears in a SQUID placed a micron away. For a SQUID loop of micron dimensions, the corresponding flux variation $\delta \Phi \sim 10^{-7} \Phi_{0}$ (with $\Phi_{0}$ a flux quantum) - within reach of modern SQUIDs. For such a setup with $\left(T_{1} / T_{0}\right) \sim 10^{-1}$, typical critical Josephson current $J_{S}^{(0)} \sim 10 \mu \mathrm{A},|\Delta|=1.0 \mathrm{meV}$, and $e V \sim 10^{-3}|\Delta|$, we find that $\alpha \sim 0.1$. As $n_{x}=\sin \theta \cos \phi$, with a similar relation for $n_{y}$, the spin components orthogonal to $\mathbf{B}$ vary, to first order in $\alpha$, with a Fourier component at frequency $\left|\omega_{L} \pm \omega_{J}\right|$, leading to a discernable signal in the magnetic field $(\mathbf{B}+\delta \mathbf{B})$. For a field $B \sim 200$ Gauss, $\omega_{L} \sim 560 \mathrm{MHz}$ wherein for a substantial voltage $\left(\omega_{J}\right)$ range, a new side band will appear at $\left|\omega_{L}-\omega_{J}\right|$ whose magnitude may be tuned to $\mathcal{O}(10-100) \mathrm{MHz}$. This measurable frequency is markedly different from that associated with standard Larmor frequency $\left(\omega_{L}\right)$ precessions.

Conclusion. By analyzing WZWN phases within the non-equilibrium Keldysh framework, we find novel nonplanar spin dynamics in the presence of a tunneling Josephson current. The coupling of the spin to a superconducting bath produces non-damping spin retardation. This retardation results from additional non-dissipative terms in the spin equations of motion. There are impor- tant differences with the case of Josephson effect in the presence of a vibrational mode 15]. The normalization of the spin disallows resonance effects at $\omega_{L}=\omega_{J}$. The polar oscillations do not lead to additional harmonics in the Josephson current. Nevertheless, the dynamics may be monitored by SQUID measurements.

We thank Yu. Makhlin for useful discussions. This work was supported by the US DOE.

[1] J. Koehler et al., Nature 363, 342 (1993); J. Wrachtrup et al., ibid. 363, 244 (1993); Phys. Rev. Lett. 71, 3565 (1993).

[2] H.-A. Engel and D. Loss, Phys. Rev. Lett. 86, 4648 (2001); Phys. Rev. B 65, 195321 (2002).

[3] Y. Manassen et al., Phys. Rev. Lett. 62, 2531 (1989); D. Shachal and Y. Manassen, Phys. Rev. B 46, 4795 (1992); Y. Manassen, J. Magnetic Reson. 126, 133 (1997); Y. Manassen, I. Mukhopadhyay, and N. Ramesh Rao, Phys. Rev. B 61, 16223 (2000).

[4] C. Durkan and M. E. Welland, Appl. Phys. Lett. 80, 458 (2002).

[5] H. Manoharan, Nature 416, 24 (2002); H. Manoharan, C. P. Lutz, and D. Eigler, Nature 403, 512 (2000).

[6] A. V. Balatsky and I. Martin, Quan. Inform. Process. 1, 53 (2002); A. V. Balatsky, Y. Manassen, and R. Salem, Phys. Rev B 66, 195416 (2002).

[7] D. Mozyrsky and I. Martin, Phys. Rev. Lett. 89, 018301 (2002).

[8] J.-X. Zhu and A. V. Balatsky, Phys. Rev. B 67, 174505 (2003).

[9] Y. Avishai, A. Golub, and A. D. Zaikin, Phys. Rev. B 63, 134515 (2001).

[10] V. Ambegaokar and A. Baratoff, Phys. Rev. Lett. 10, 486 (1963); 11, 104(E) (1963).

[11] M. Sigrist and K. Ueda, Rev. Mod. Phys. 63, 239 (1991); V. Geshkenbein and A. I. Larkin, JETP Lett. 43, 395 (1986); D. A. Wollman et. al., Phys. Rev. Lett. 71, 2134 (1993).

[12] U. Eckern, G. Schön, and V. Ambegaokar, Phys. Rev. B 30, 6419 (1984); A.I. Larkin and Yu. N. Ovchinnikov, Phys. Rev. B 28, 6281 (1983).

[13] E. Fradkin, Field Theories of Condensed Matter Systems (Addison-Wesley, Redwood City, 1991); S. Sachdev, Quantum Phase Transitions (Cambridge University Press, London, 1999).

[14] These two orthogonal AF fields represent (i) the slowly varying staggered spin field (the antiferromagnetic staggered moment $\mathbf{m}$ taking on the role of $\mathbf{S}_{1}$ ) and (ii) the rapidly oscillating uniform spin field $\mathbf{l}$ (paralleling our $\mathbf{S}_{2}$ ). In the antiferromagnetic correspondence, the two forward time spin trajectories at two nearest neighbor AF sites become the two forward $(u)$ and backward $(l)$ single spin trajectories of the non-equilibrium problem. This staggered doubling correspondence is general.

[15] J.-X. Zhu, Z. Nussinov, and A. V. Balatsky, cond-mat/0306107 